\title{
How I deploy arterial grafts
}

\section{David P. Taggart}

John Radcliffe Hospital, University of Oxford, Oxford OX3 9DU, UK

Correspondence to: David P. Taggart, MD(Hons), PhD, FRCS, FESC. Professor of Cardiovascular Surgery, John Radcliffe Hospital, University of Oxford, Oxford OX3 9DU, UK. Email: David.taggart@ouh.nhs.uk.

\begin{abstract}
There are currently around one million coronary artery bypass graft (CABG) procedures performed worldwide annually and despite two decades of evidence to support the use of a second arterial graft, if not total arterial grafting, the stark reality is that in contemporary practice $80 \%$ of all grafts used for CABG are saphenous vein grafts (SVG). The following description of how I deploy arterial grafts has been developed over more than two decades of clinical practice and largely self-taught by a process of "trial and error" and most importantly dictated by "ease of use" rather than personal robust angiographic data of long-term patency (although there is other such existing data in the literature for some of these techniques). The fact that there are numerous potential variants of deploying multiple arterial grafts underpins the fact that there is no single operation that is ideal in every patient-it is not a case of "one size fits all" as would be the situation for the use of a single internal thoracic artery (ITA) and supplemental vein grafts.
\end{abstract}

Keywords: Internal thoracic artery (ITA); radial artery (RA); off-pump; coronary artery bypass graft (CABG)

Submitted Sep 18, 2018. Accepted for publication Sep 20, 2018.

doi: $10.21037 /$ acs.2018.09.06

View this article at: http://dx.doi.org/10.21037/acs.2018.09.06

\section{Introduction}

In terms of grafts used in addition to the left internal thoracic artery (LITA), the majority of evidence focuses on use of the right ITA (RITA) or radial artery (RA). In the West the RA is largely used as a second or third arterial graft while the use of the gastroepiploic artery (GEA) remains common by some surgeons in the Far East.

While there is a very substantial observational literature supporting the use of bilateral ITA (BITA) grafts (1-4), there has only been one large randomized controlled trial (RCT) (5) that has attempted to answer this. The longawaited 10 years results of the Arterial Revascularisation Trial (ART) were presented on behalf of the ART Investigators at the ESC in Munich 2018 (6). At the time of the conception of ART in 2002 the prevailing wisdom was that it was only the use of bilateral ITA (BITA) grafts that might potentially offer an additional survival benefit to a single LITA graft. At 10 years, in the randomized intention-to-treat analysis in ART there was no difference in mortality between BITA and LITA groups, nor in the composite endpoints of mortality, myocardial infarction (MI) or stroke. In contrast, in the as-treated analysis (non-randomized), performed because $40 \%$ of patients effectively received a different treatment strategy from that originally intended [14\% of BITA received a single LITA, $4 \%$ of single ITA (SITA) received BITA and $22 \%$ of SITA received an RA], there was a significant survival benefit (and a significant reduction in the composite endpoint) in favour of more than one arterial graft. In the ESC presentation, data was provided to show that surgeon experience was critical to successfully perform BITA grafting and that high performers had superior surgical outcomes at 10 years.

Recently, the long-debated question of whether the use of a RA is superior to saphenous vein grafts (SVG) has been answered conclusively by the RADIAL Investigators in a meta-analysis of six randomized trials with 5-year followup (7). Briefly, a lower angiographic failure rate of the RA vs. SVG ( $8 \%$ vs. 20\%) accompanied by a significant reduction in the composite end-point of death, MI and repeat revascularization definitively settles the question. Furthermore, with continuing attrition of vein grafts over 
10 years it could be speculated that the differences in patency and clinical outcome may continue to diverge even further in favour of the RA.

\section{General considerations in deploying arterial grafts}

In deploying arterial grafts to give the best long-term chance of success in combination with their "ease of use" there are six major considerations:

(I) The putative risk of competitive flow in the native coronary artery. This usually has to be estimated visually, as fractional flow reserve (FFR) measurements (that give a far more accurate estimate of the real functional significance of any particular coronary artery stenoses) are not routinely available. Generally arterial grafts should not be used unless the native coronary artery stenoses is greater than $70 \%$. The ITA graft may be more resistant to competitive flow than the RA;

(II) There is increasing acceptance that as a consequence of their well documented superior long-term angiographic patency, more arterial grafts should, in general, be used in younger patients (probably now defined as less than 70 years old), especially with good ventricular function and absence of life-limiting co-morbidity. It is my opinion that more arterial grafts should also be more widely used in the elderly to allow a true "no touch aortic technique" where there is robust evidence for a reduction in the risk of most major complications and, in particular, stroke (8);

(III) In deciding whether to use a second ITA graft or the RA the risk of mediastinitis needs to be considered (9). The highest risk groups are those with insulin dependent diabetes mellitis, obesity, female gender, poor respiratory function and the need for immunosuppressants/steroids. Any combination of these factors increases the risk of sternal wound complications even when harvesting ITA grafts in a skeletonized fashion;

(IV) For arterial grafts to the left side, using composite arterial grafts based on the LITA (i.e., with RITA, RA or SVG) both allows more distal anastomoses than an in situ RITA and eliminates the potential major hazard of damage to the RITA during redo sternotomy. Furthermore, if using the RITA as a composite graft from the medial side of the LITA only the proximal two/thirds of the RITA needs to be harvested to provide adequate length even for sequential grafting;

(V) When constructing composite grafts a " $\mathrm{T}$ " anastomoses is less likely to kink than a "Y" anastomoses-especially on the lateral wall of the heart;

(VI) Use of multiple arterial grafts, especially when performed off-pump, may have significant clinical benefits in specific situations but is a much more technically challenging procedure than the use of a single LITA graft and supplemental vein grafts performed on pump. Consequently, this author believes that it is mandatory to confirm that all grafts are patent before the patient leaves the operating room as a technically failed graft will frequently not immediately result in haemodynamic, ECG or echocardiographic changes but may do so subsequently (10). The author considers it essential to "prove that you did a great job" rather than to think it. Additionally if a proximal anastomosis is required to the ascending aorta then ultrasound imaging should be used to identify a truly disease-free area of aorta. Even the most perceptive finger cannot identify soft atheromatous plaque on the luminal side of the aorta.

\section{General preparation for graft deployment}

This is dictated by two considerations. First it depends whether the operation is performed on or off-pump. In the former situation the sequence of distal graft anastomoses is less relevant and usually LITA to left anterior descending (LAD) is the last distal anastomoses. For off-pump CABG, however, the over-riding principle is that any occluded coronary artery must be revascularized first.

Second it depends whether a composite arterial graft is to be performed. If so, it is our usual practice to construct a RITA to the medial side of LITA as a "T" anastomoses on a swab placed over the ascending aorta at the level of the second intercostal space where there is relatively little movement.

For off-pump procedures it is our usual practice to first perform LITA grafting to the lateral wall, followed by grafting of the inferior wall and finally grafting to the LAD. Grafting the LAD first can cause uncomfortable stretching 


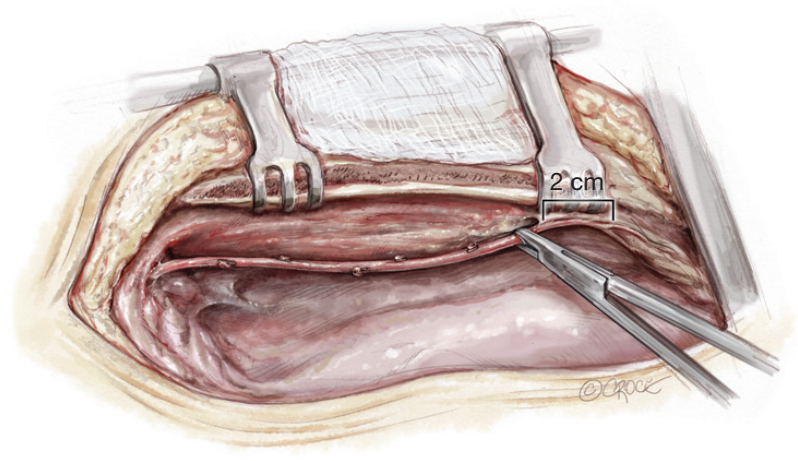

Figure 1 Skeletonized harvest of the ITA. ITA, internal thoracic artery.

and tension on a LITA graft while displacing the heart into the right chest cavity to expose the lateral wall.

When performing the procedure off-pump our practice is to:

(I) Open both pleurae widely;

(II) Divide the pericardium on the right side to the inferior vena cava so that the heart can easily be placed in the right chest to expose the lateral wall;

(III) Divide the pericardium on the left side towards the phrenic nerve at the second intercostal space to avoid tension on left sided arterial grafts to the lateral wall;

(IV) Perform a cruciate incision in the posterior pericardium below the phrenic nerve which can be palpated with the left hand (when the pericardium is tensed) to effectively eliminate the incidence of pericardial tamponade [and possibly post-operative atrial fibrillation (11)].

\section{General strategy for graft deployment}

The following attempts to describe potential advantages and disadvantages of various techniques for deploying arterial grafts in differing anatomical situations and useful 'tips and "tricks".

\section{Figure 1: Skeletonized harvest of the ITA}

Skeletonization of the ITA results in a conduit that is both longer and wider that makes composite and sequential grafting easier and also significantly reduces the incidence of sternal wound complications when BITA grafts are used (12). To harvest the LITA by the skeletonisation technique, the intra-thoracic fascia medial to the LITA is incised as a "tram line" with diathermy or scissors. The ITA vein lies medial to the ITA and is used as an anatomic guide to safe harvest of the ITA. We use small clips on each ITA branch proximally and distally and then divide each branch with fine scissors. Some surgeons favour the use of a harmonic scalpel to harvest the ITA and avoid the need for multiple clips (13).

The ITA harvest stops 2 centimetres proximal to the bifurcation, both to avoid the risk of devascularisation of the xiphisternum and because this portion of the artery is known to be more vasospastic than the more proximal LITA (14). During harvest there is no need to heparinize even after dividing the ITA from the chest wall and occluding it distally with a clip. To encourage ITA vasodilatation, systolic blood pressure is increased to $150 \mathrm{mmHg}$ for 5 minutes without the need for topical vasodilators. In a small number of patients where the ITA diameter appears very small (usually small elderly females) it may be necessary to carefully inject a small amount of papaverine diluted in blood intraluminally. This must be done with a very fine catheter to avoid iatrogenic injury to the artery.

\section{Figure 2: In situ RITA to LAD and LITA to circumflex system}

The advantage of this technique is its technical simplicity for both on and off-pump CABG. It is particularly useful in the setting of distal left main coronary artery disease, with or without additional proximal left sided disease, and facilitates a no touch aortic technique to minimize the risk of stroke, particularly in elderly patients.

\section{Figure 3: LITA to an obtuse marginal with a composite RITA as a free graft to the LAD}

The major advantage is its technical simplicity especially when performed off-pump. It is technically less challenging than bringing the RITA from the lateral wall of the LITA to the obtuse marginal system. Furthermore, it only requires harvest of the proximal two thirds of the RITA.

\section{Figure 4: Composite sequential RITA from LITA}

Composite sequential arterial grafts on the left side with 


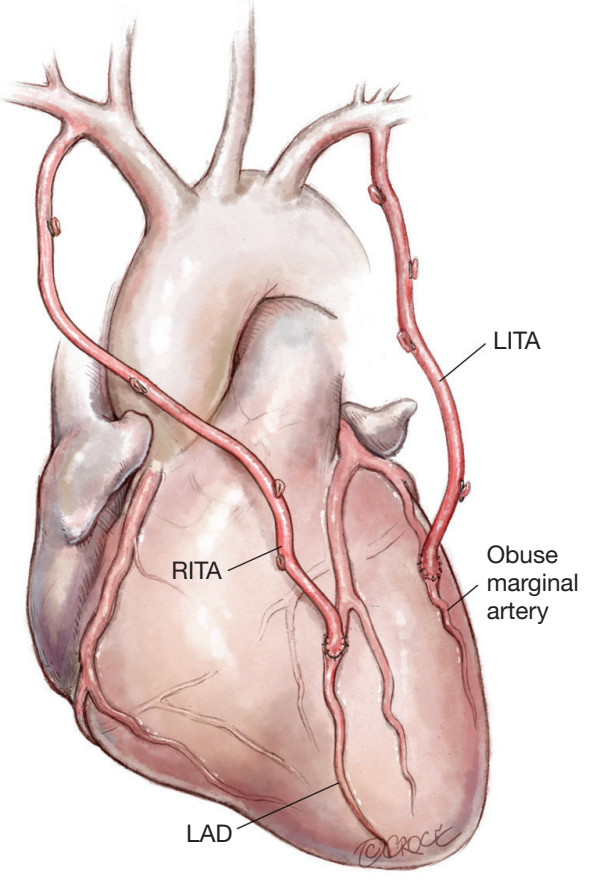

Figure 2 In situ RITA to LAD and LITA to circumflex system. RITA, right internal thoracic artery; LITA, left internal thoracic artery; LAD, left anterior descending.

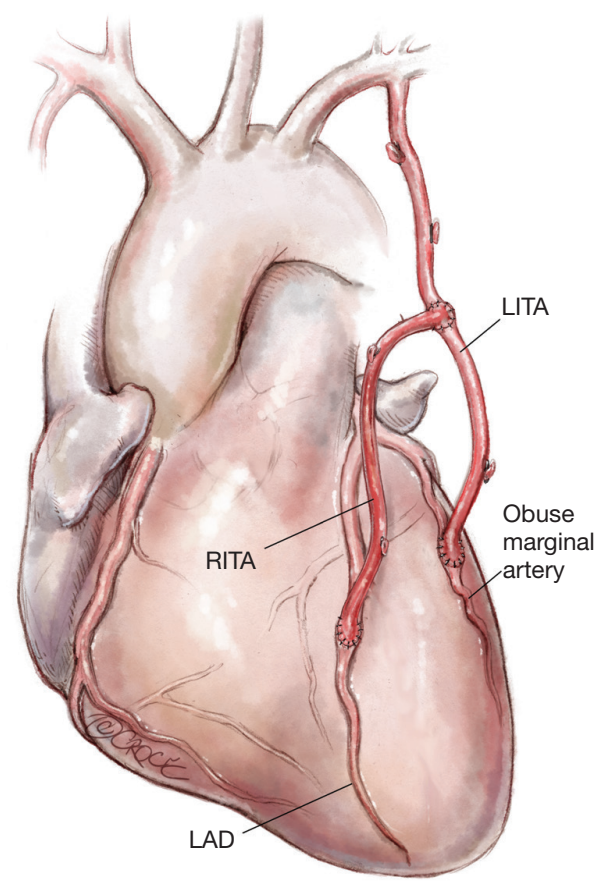

Figure 3 LITA to an obtuse marginal with a composite RITA as a free graft to the LAD. RITA, right internal thoracic artery; LITA, left internal thoracic artery; LAD, left anterior descending.

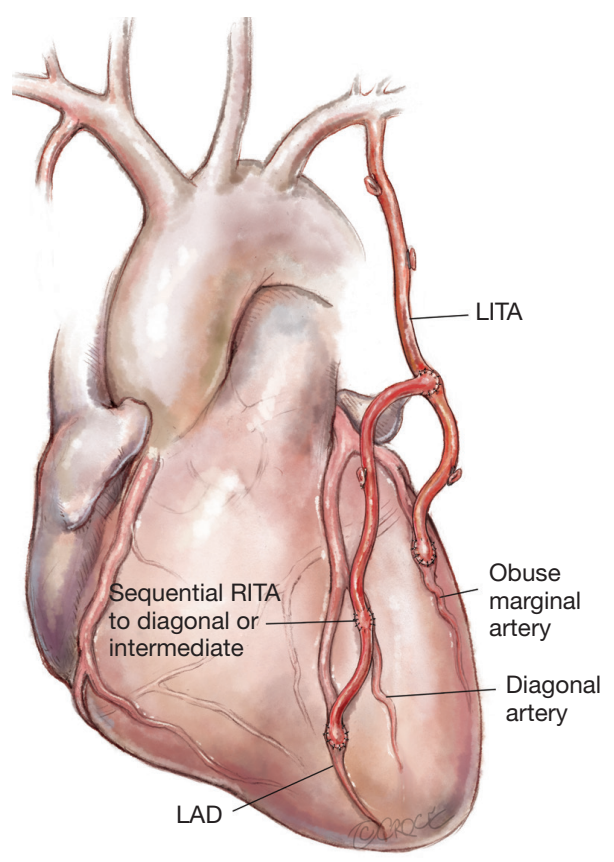

Figure 4 Composite sequential RITA from LITA. RITA, right internal thoracic artery; LITA, left internal thoracic artery; LAD, left anterior descending.

the RITA anastomosed as a "T" graft from the medial side of the LITA and then used sequentially to pick up an intermediate and/or a diagonal coronary artery as a side-to-side anastomosis before end-to-side anastomosis to the LAD. The author feels that this is less technically demanding than using the RITA as a composite graft from the lateral wall of the LITA to perform sequential composite grafts on the lateral wall of the heart. In off-pump surgery, if a LITA graft to the obtuse marginal coronary artery (OM) is performed first then the heart can be then placed back in the pericardial cavity and the lie of the RITA coming from the medial side of the LITA back towards the midline, picking up an intermediate and/or diagonal coronary artery as a side-to-side anastomosis, is technically more straightforward and results in an excellent lie of the grafts.

\section{Figure 5: Composite RITA from lateral wall of LITA with additional "T graft"}

A LITA conduit (to the LAD) with a composite RITA conduit from its lateral wall. The RITA conduit has an additional conduit (recycled ITA/RA/SVG) performed as a "T" graft for further grafting on the lateral/posterolateral wall. 


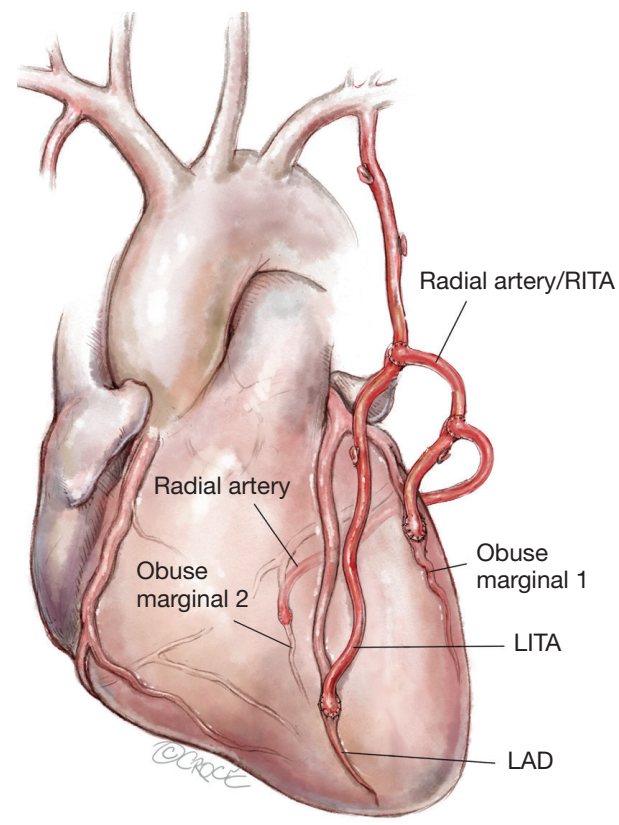

Figure 5 Composite RITA from lateral wall of LITA with additional "T graft". RITA, right internal thoracic artery; LITA, left internal thoracic artery; LAD, left anterior descending.

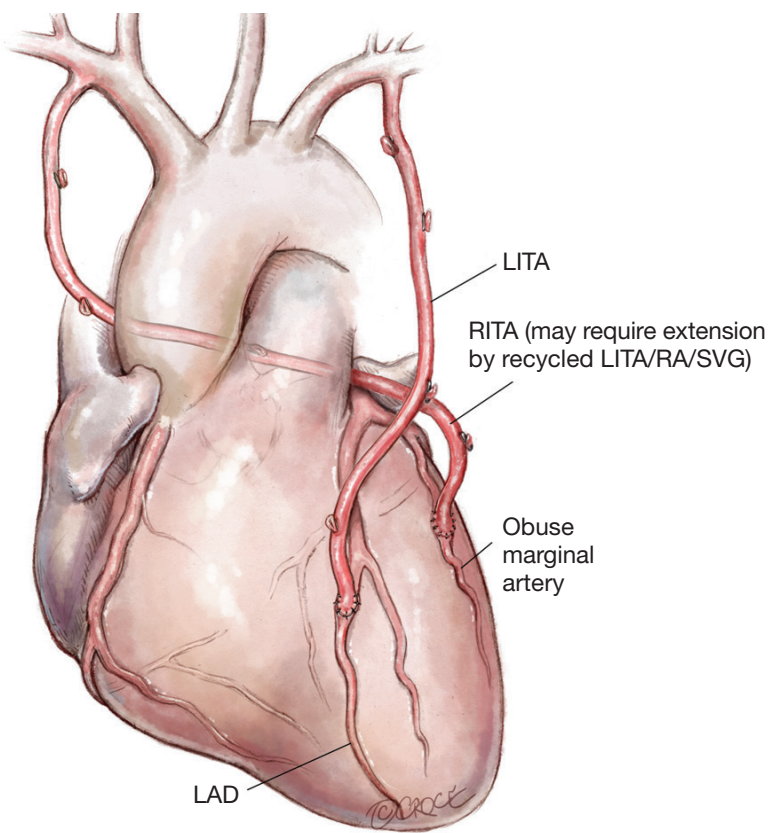

Figure 6 LITA to LAD, RITA through the transverse sinus to the obtuse marginal. RITA, right internal thoracic artery; LITA, left internal thoracic artery; LAD, left anterior descending; RA, radial artery; SVG, saphenous vein graft.

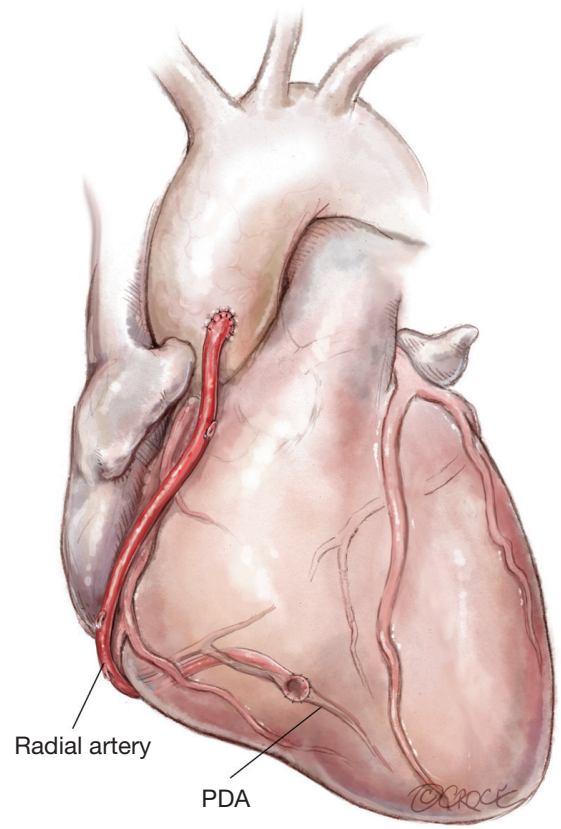

Figure 7 Radial artery from aorta to PDA. PDA, posterior descending artery.

\section{Figure 6: LITA to LAD, RITA through the transverse sinus to the obtuse marginal}

The advantage of this technique is to avoid any arterial graft crossing the mid line in case of eventual need for redo sternotomy. There are two disadvantages of this technique. First is the unusual scenario of bleeding from a side branch of the RITA behind the aorta. Second and more commonly the RITA can only reach early intermediate or obtuse marginal artery and may appear stretched when performed off pump. In such a scenario the RITA may require extension by recycled LITA/RA/SVG.

\section{Figure 7: RA from aorta to posterior descending artery (PDA)}

This is an excellent strategy where there is at least $80 \%$ stenosis in the right coronary artery (RCA) or preferably if the RCA is occluded when competitive flow is minimized. An alternative strategy, especially if a no touch aortic technique is indicated, is to anastomose the RA to the proximal in situ RITA with the main body of the RITA being used for a composite left sided graft. 


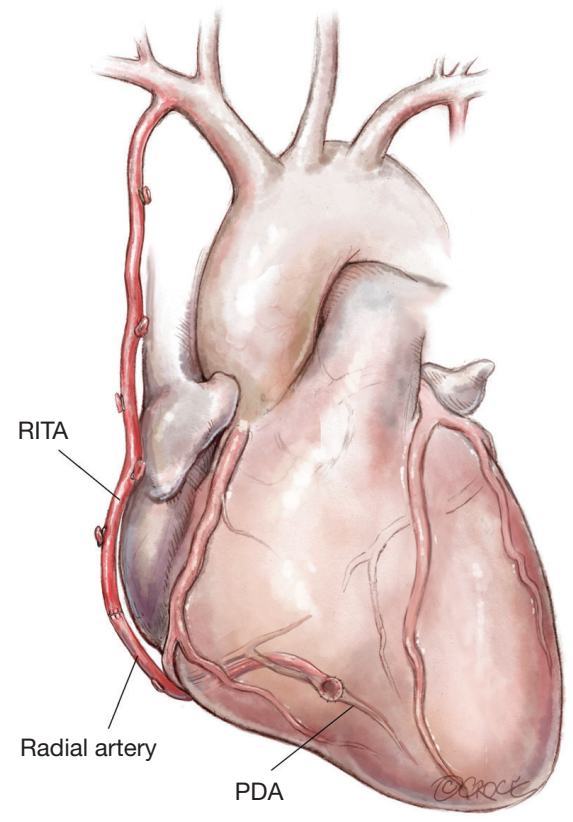

Figure 8 In situ RITA with extension by radial artery. RITA, right internal thoracic artery; PDA, posterior descending artery.

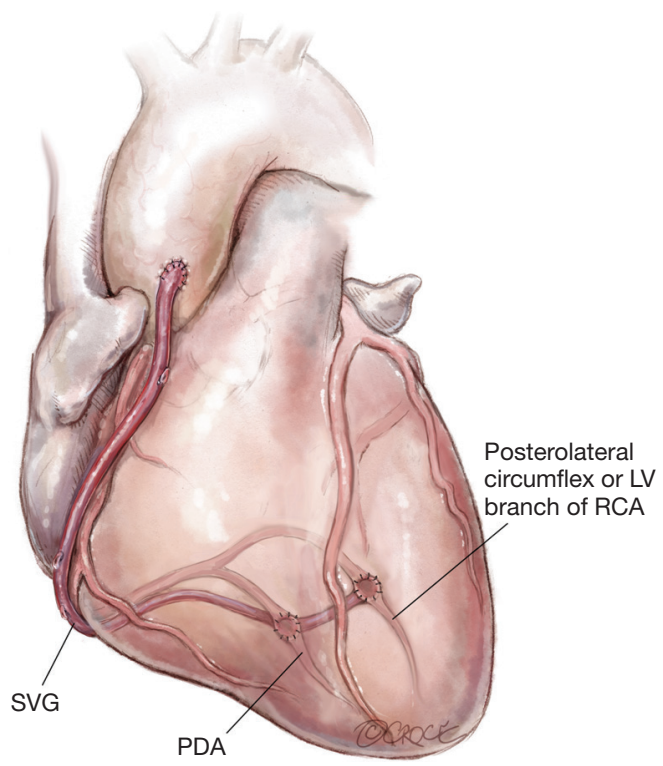

Figure 9 Sequential saphenous vein graft on the inferior wall of the heart. LV, left ventricular; RCA, right coronary artery; SVG, saphenous vein graft; PDA, posterior descending artery.

\section{Figure 8: In situ RITA with extension by RA}

The RITA will commonly not reach the PDA even after full skeletonization and can be extended with recycled LITA/RA/SVG. The RITA should not be placed to the main RCA because of the potential for competitive flow due to size discrepancy and eventual development of progressive disease at the crux. As described for Figure 7, an alternative strategy is to anastomose the RA or SVG to the proximal in situ RITA, especially if a no touch aortic technique is indicated, with the main body of the RITA being used for a composite left sided graft.

\section{Figure 9: Sequential SVG on the inferior wall of the} heart

This technique uses a SVG with sequential side-toside anastomosis to the PDA of the right coronary and then end-to-side to a significant left ventricular (LV) coronary artery on the inferior wall either from the right or from the left coronary system. This is a technically straightforward sequential graft with an excellent lie. It is usually easier to perform the side-to-side anastomosis to the PDA before either a side-to-side or end-to-side anastomosis to the $\mathrm{LV}$ branch.

\section{Figure 10: Use of a GEA for the inferior wall}

The use of a GEA to the PDA with an in situ RITA to the LAD and LITA to the obtuse marginal. GEA to the PDA is particularly useful in the unusual situation of an occluded dominant ungrafted/failed graft to the right coronary artery but with patent grafts to the left side. This can be performed off pump through a relatively limited incision through the lower sternum.

\section{Figure 11: RA jump graft from distal LAD to proximally occluded PDA}

LITA to LAD with a jump graft from the distal LAD to the PDA. This is technically easy to do off pumppreserving the no touch aortic technique principal and proving useful in the situation when there is limited conduit. 


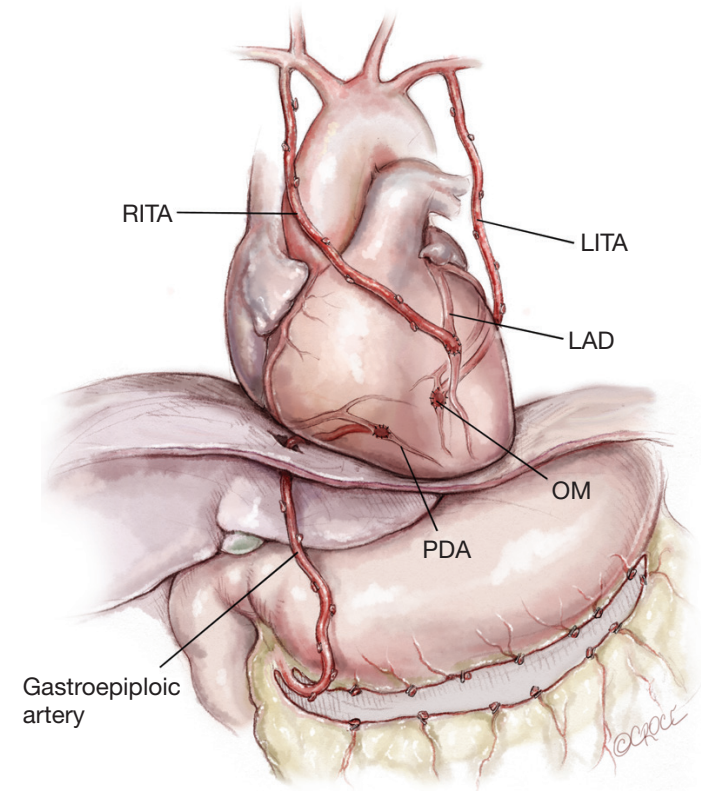

Figure 10 Use of a gastroepiploic artery (GEA) for the inferior wall. RITA, right internal thoracic artery; LITA, left internal thoracic artery; PDA, posterior descending artery; OM, obtuse marginal coronary artery; LAD, left anterior descending.

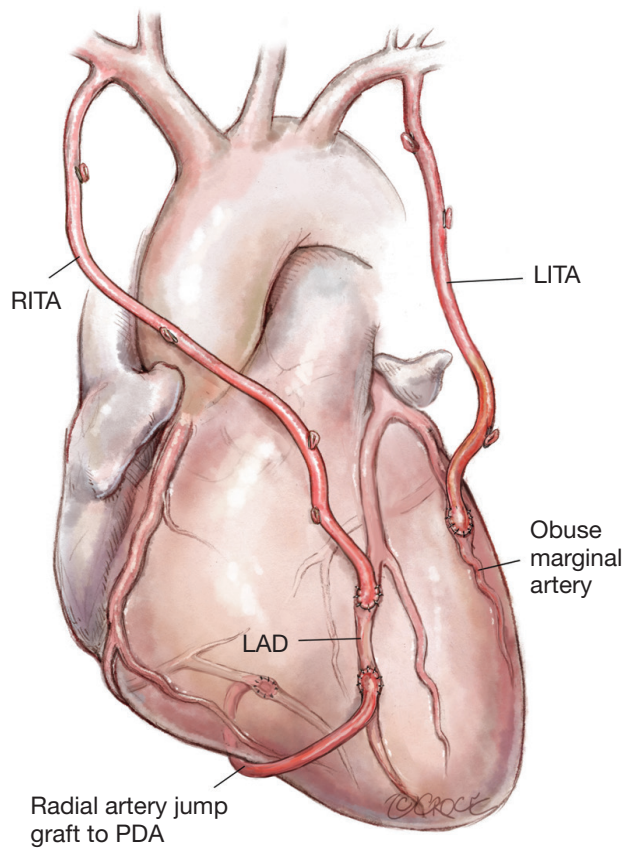

Figure 11 Radial artery jump graft from distal LAD to proximally occluded PDA. RITA, right internal thoracic artery; LITA, left internal thoracic artery; LAD, left anterior descending; PDA, posterior descending artery.

\section{Acknowledgements}

None.

\section{Footnote}

Conflicts of Interest: Prof. DP Taggart has received research funding, speaking and travelling honoraria from Medistim (Oslo) and is the Principle Investigator for the REQUEST Registry (funded by Medistim).

\section{References}

1. Taggart DP, D'Amico R, Altman DG. Effect of arterial revascularisation on survival: a systematic review of studies comparing bilateral and single internal mammary arteries. Lancet 2001;358:870-5.

2. Weiss AJ, Zhao S, Tian DH, et al. A meta-analysis comparing bilateral internal mammary artery with left internal mammary artery for coronary artery bypass grafting. Ann Cardiothorac Surg 2013;2:390-400.

3. Yi G, Shine B, Rehman SM, et al. Effect of bilateral internal mammary artery grafts on long-term survival: a meta-analysis approach. Circulation 2014;130:539-45.

4. Buttar SN, Yan TD, Taggart DP, et al. Long-term and short-term outcomes of using bilateral internal mammary artery grafting versus left internal mammary artery grafting: a meta-analysis. Heart 2017;103:1419-26.

5. Taggart DP, Altman DG, Gray AM, et al. Randomized Trial of Bilateral versus Single Internal-Thoracic-Artery Grafts. N Engl J Med 2016;375:2540-9.

6. Taggart DP. 10 year outcomes of the ART trial. ESC Hotline Session Munich 2018. [In press].

7. Gaudino M, Benedetto U, Fremes S, et al. Radial-Artery or Saphenous-Vein Grafts in Coronary-Artery Bypass Surgery. N Engl J Med 2018;378:2069-77.

8. Zhao DF, Edelman JJ, Seco M, et al. Coronary Artery Bypass Grafting With and Without Manipulation of the Ascending Aorta: A Network Meta-Analysis. J Am Coll Cardiol 2017;69:924-36.

9. Gaudino M, Taggart D, Suma H, et al. The Choice of Conduits in Coronary Artery Bypass Surgery. J Am Coll Cardiol 2015;66:1729-37.

10. Amin S, Pinho-Gomes AC, Taggart DP. Relationship of Intraoperative Transit Time Flowmetry Findings to Angiographic Graft Patency at Follow-Up. Ann Thorac Surg 2016;101:1996-2006.

11. Asimakopoulos G, Della Santa R, Taggart DP. Effects 
of posterior pericardiotomy on the incidence of atrial fibrillation and chest drainage after coronary revascularization: a prospective randomized trial. J Thorac Cardiovasc Surg 1997;113:797-9.

12. Benedetto U, Altman DG, Gerry S, et al. Pedicled and skeletonized single and bilateral internal thoracic artery grafts and the incidence of sternal wound complications: Insights from the Arterial Revascularization Trial. J
Thorac Cardiovasc Surg 2016;152:270-6.

13. Kieser TM, Rose MS, Aluthman U, et al. Quicker yet safe: skeletonization of 1640 internal mammary arteries with harmonic technology in 965 patients. Eur J Cardiothorac Surg 2014;45:e142-50.

14. He GW, Taggart DP. Spasm in Arterial Grafts in Coronary Artery Bypass Grafting Surgery. Ann Thorac Surg 2016;101:1222-9.

Cite this article as: Taggart DP. How I deploy arterial grafts. Ann Cardiothorac Surg 2018;7(5):690-697. doi: 10.21037/ acs.2018.09.06 